\title{
On the perspectives of using XMM to study fundamental parameters of early-type stars
}

\author{
Gregor Rauw ${ }^{1,6}$, Karel A. van der Hucht ${ }^{2}$, Rolf Mewe $^{2}$, \\ Manuel Güdel ${ }^{3}$, Jean-Marie Vreux ${ }^{1}$, Eric Gosset ${ }^{1,6}$, Werner Schmutz ${ }^{4}$ \\ and Ian R. Stevens ${ }^{5}$ \\ ${ }^{1}$ Institut d'Astrophysique et de Géophysique, Liège, Belgium \\ ${ }^{2}$ Space Research Organization Netherlands, Utrecht, the Netherlands \\ ${ }^{3}$ ETHZ, Paul Scherrer Institute, Villingen, Switzerland \\ ${ }^{4}$ ETH, Zürich, Switzerland \\ ${ }^{5}$ School of Physics \& Astronomy, Birmingham, United Kingdom
}

\section{Introduction}

Although substantial progress has been achieved since the discovery of X-ray emission from early-type stars with the EINSTEIN satellite, several crucial aspects of this phenomenon are still not fully understood. Considerable breakthroughs in this field are expected from observations with the X-ray Multi-Mirror satellite $(X M M)$ due for launch in early 2000. $X M M$ is the second cornerstone mission of the ESA Horizon 2000 science programme (see Lumb et al. 1996 and references therein for an overall description of the satellite). XMM offers a large effective area over a wide range of energies and its instrumentation provides simultaneously non-dispersive spectroscopic imaging (EPIC - European Photon Imaging Camera), medium-resolution dispersive spectroscopy (RGS - Reflection Grating Spectrometer) and optical-UV imaging (OM - Optical Monitor).

\section{Simulated $X M M$ spectroscopy}

In the present paper, we highlight some of the expected scientific contributions of XMM to massive star research. All the simulations were performed with the SPEX-code (Kaastra et al. 1996).

First, we have simulated a $50 \mathrm{ksec} X M M$-RGS exposure of the WN7+abs star WR 25 assuming various chemical compositions of the WR wind. To illustrate the potential of actual RGS data to perform abundance studies, the fake spectra were rebinned to achieve a $\mathrm{S} / \mathrm{N} \geq 3$ in each bin and were then fitted keeping the column densities fixed. For instance, our simulated RGS spectra allow us to recover the abundances of $\mathrm{O}$ and $\mathrm{Ne}$ of the input models to $\sim 10-15 \%$. Our results therefore indicate that $X M M$ will give access to consistent abundance measurements on key elements which are difficult or impossible to constrain from longer wavelength observations, such as $\mathrm{Fe}, \mathrm{O}, \mathrm{Ne}$ and $\mathrm{Mg}$ (RGS), but also $\mathrm{Si}$ and S (EPIC). These abundance measurements are crucial for our understanding of stellar evolution in the WR phase.

\footnotetext{
${ }^{6}$ At the Fonds National de la Recherche Scientifique, Belgium
} 


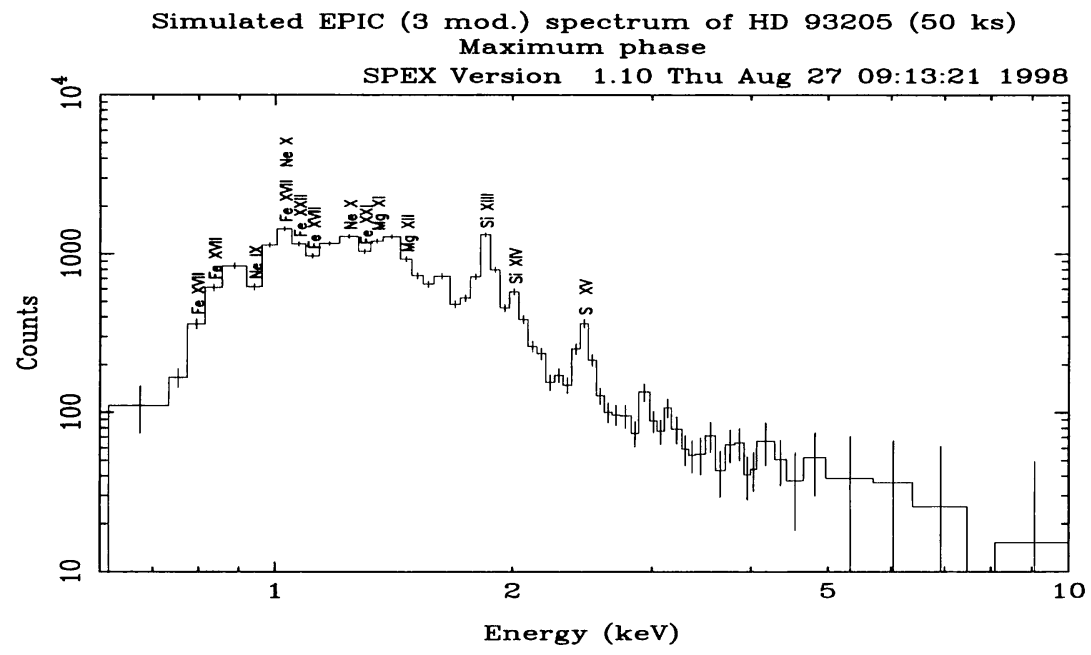

Figure 1. Simulated $50 \mathrm{ksec}$ XMM-EPIC exposure of HD 93205.

It is worth noticing that XMM observations of WR 25 will provide simultaneously EPIC data on most of the stars in the $\operatorname{Tr} 14$ and $\operatorname{Tr} 16$ open clusters, including the close binary HD $93205(\mathrm{O} 3 \mathrm{~V}+\mathrm{O} 8 \mathrm{~V})$. ROSAT-PSPC observations of HD 93205 reveal phase-locked variations characteristic of a colliding wind system (Corcoran 1996). Figure 1 illustrates a simulated XMM-EPIC spectrum of HD 93205. The parameters used for this system were adapted from the two temperature model fitted by Pittard \& Stevens (1997) to their maximum flux synthetic spectrum of HD 57060. We assume solar abundances and the emission measures are scaled according to the corresponding ROSAT-PSPC count rates and adopting a distance of $3.1 \mathrm{kpc}$ for HD 93205. Thanks to the high sensitivity of $X M M$, the quality of the spectrum of HD 93205 will allow a quantitative comparison with model predictions from hydrodynamical simulations (Pittard \& Stevens 1997). Such a comparison will provide constraints on the key properties of the winds of both components and can therefore help to assess the mass loss rates in a new independent way. Also, given the high sensitivity of XMM, we will, for the first time, be able to investigate the short timescale variability of the X-ray flux, resulting from hydrodynamical instabilities of the shock region.

\section{References}

Corcoran, M.F. 1996, in: V.S. Niemela \& N. Morrell (eds.), Collinding Winds in Binary Stars, RevMexAA-SC 5, 54

Kaastra, J.S., Mewe, R., Nieuwenhuijzen, H. 1996, in: K. Yamashita \& T. Watanabe (eds.), UV and X-ray Spectroscopy of Astrophysical and Laboratory Plasmas, (Tokyo: Univ. Acad. Press. Inc.), p. 411

Lumb, D.H., Eggel, H., Lainé, R., Peacock, A.J. 1996, in: O.H. Siegmund \& M.A. Gummin (eds.), EUV, X-ray, and Gamma Ray Instrumentation for Astronomy VII, Proc. SPIE, Vol. 2808, p. 326

Pittard, J.M., Stevens, I.R. 1997, MNRAS 292, 298 